2011

\title{
Uniform 11 Behavior of a Time Discretization Method for a Volterra Integrodifferential Equation With Convex Kernel; Stability
}

\author{
Charles B. Harris \\ Old Dominion University \\ Richard D. Noren \\ Old Dominion University
}

Follow this and additional works at: https://digitalcommons.odu.edu/mathstat_fac_pubs

Part of the Applied Mathematics Commons

\section{Repository Citation}

Harris, Charles B. and Noren, Richard D., "Uniform 11 Behavior of a Time Discretization Method for a Volterra Integrodifferential Equation With Convex Kernel; Stability" (2011). Mathematics \& Statistics Faculty Publications. 17.

https://digitalcommons.odu.edu/mathstat_fac_pubs/17

\section{Original Publication Citation}

Harris, C. B., \& Noren, R. D. (2011). Uniform $\iota^{1}$ behavior of a time discretization method for a volterra integrodifferential equation with convex kernel; stability. SIAM Journal on Numerical Analysis, 49(4), 1553-1571. doi:10.1137/100804656 
UNIFORM $l^{1}$ BEHAVIOR OF A TIME DISCRETIZATION METHOD
FOR A VOLTERRA INTEGRODIFFERENTIAL EQUATION WITH
CONVEX KERNEL; STABILITY*

CHARLES B. HARRIS ${ }^{\dagger}$ AND RICHARD D. NOREN ${ }^{\dagger}$

\begin{abstract}
We study stability of a numerical method in which the backward Euler method is combined with order one convolution quadrature for approximating the integral term of the linear Volterra integrodifferential equation $\mathbf{u}^{\prime}(t)+\int_{0}^{t} \beta(t-s) \mathbf{A} \mathbf{u}(s) d s=0, t \geq 0, \mathbf{u}(0)=\mathbf{u}_{0}$, which arises in the theory of linear viscoelasticity. Here $\mathbf{A}$ is a positive self-adjoint densely defined linear operator in a real Hilbert space, and $\beta(t)$ is locally integrable, nonnegative, nonincreasing, convex, and $-\beta^{\prime}(t)$ is convex. We establish stability of the method under these hypotheses on $\beta(t)$. Thus, the method is stable for a wider class of kernel functions $\beta(t)$ than was previously known. We also extend the class of operators $\mathbf{A}$ for which the method is stable.
\end{abstract} stability

Key words. Volterra integrodifferential equation, convolution quadrature, convex kernel, $l^{1}{ }_{-}$

AMS subject classifications. 45D05, 45K05, 65R20, 64D05

DOI. $10.1137 / 100804656$

1. Introduction. Let $\mathbf{A}$ be a positive self-adjoint linear operator defined on a dense subspace $\mathcal{D}(\mathbf{A})$ of a real Hilbert space $\mathbf{H}$ with spectral decomposition

$$
\mathbf{A x}=\int_{-\infty}^{\infty} \lambda d \mathbf{E}_{\lambda} \mathbf{x}
$$

for $\mathbf{x} \in \mathcal{D}(\mathbf{A})$. We assume that the spectrum of $\mathbf{A}$ is contained in $\left[\lambda_{0}, \infty\right)$, where $\lambda_{0}>0$. Xu established stability results in 2002 (see [21]) and convergence results in 2008 (see [22]) for a numerical method for approximating the initial value problem

$$
\mathbf{u}^{\prime}(t)+\int_{0}^{t} \beta(t-s) \mathbf{A u}(s) d s=0, \quad t \geq 0, \quad \mathbf{u}(0)=\mathbf{u}_{0} .
$$

Here $\mathbf{u}=\mathbf{u}(t)$ is a function in the Hilbert space $\mathbf{H}$ and ${ }^{\prime}=d / d t$. Xu assumes in both papers that the kernel $\beta(t):(0, \infty) \rightarrow \mathbb{R}$ satisfies

$$
\beta \in C(0, \infty) \cap L^{1}(0,1) \text { and } 0 \leq \beta(\infty)<\beta(0+) \leq \infty,
$$

and

$$
(-1)^{n} \beta^{(n)}(t) \geq 0, \quad t>0, \quad n=0,1,2, \ldots
$$

In Theorems 1 and 2 we substantially enlarge the class of functions $\beta(t)$ for which the stability results are valid by weakening the completely monotone hypotheses (4) on $\beta(t)$ to the assumption

$$
\beta \text { is nonnegative, nonincreasing, convex, and }-\beta^{\prime} \text { is convex. }
$$

\footnotetext{
*Received by the editors August 6, 2010; accepted for publication (in revised form) May 25, 2011; published electronically July 28, 2011.

http://www.siam.org/journals/sinum/49-4/80465.html

${ }^{\dagger}$ Department of Mathematics and Statistics, Old Dominion University, Norfolk, VA 23529 (charr084@odu.edu, rnoren@odu.edu).
} 
We also note that our results hold for the wider class of operators $\mathbf{A}$ defined via a spectral family $\left\{\mathbf{E}_{\lambda}\right\}$, as in (1), whereas [21] employed the more restrictive condition that $\mathbf{A}$ possess a countable complete eigensystem.

$\mathrm{Xu}$ utilized a discrete analogue of the Payley-Wiener theorem in [23] to obtain results similar to those in the present paper for a class of quadratures and for certain kernels displaying log convexity. Although the hypotheses in [23] overlap ours, our results hold for kernels lacking log convexity, such as if $\beta(t)=0$ for some $t>0$. As an example,

$$
f(x)= \begin{cases}\left(x_{0}-t\right)^{2} & \text { for } 0 \leq x \leq x_{0} \\ 0 & \text { for } x_{0}<x\end{cases}
$$

for any fixed $x_{0}>0$.

Denote the Laplace transform of a function $f$ by $\widehat{f}(t)$. Thus,

$$
\widehat{\beta}(t)=\int_{0}^{\infty} e^{-t s} \beta(s) d s, \quad t>0 .
$$

By Bernstein's theorem [20, Chapter 8], a function $a=a(t)$ is completely monotonic iff there exists an associated nonnegative, increasing function $\alpha:[0, \infty) \rightarrow[0, \infty)$ with

$$
a(t)=\int_{0}^{\infty} e^{-x t} d \alpha(x), \quad t>0 .
$$

From (7) we see that the Laplace transform of $a(t)$ may be analytically extended to the slit plane $\mathbb{C}^{\prime} \equiv \mathbb{C} \backslash(-\infty, 0]$ via the formula

$$
\widehat{a}(t)=\int_{0}^{\infty} \frac{d \alpha(s)}{s+t} \quad t \in \mathbb{C}^{\prime}
$$

Here a Stieltjes integral is used. Xu makes extensive use of this representation in his analysis.

A convex function will only be guaranteed to have a second derivative almost everywhere [18, Chapter 7]. In particular, the representation (8) does not hold. Without this representation we are still able to obtain the same conclusions as $\mathrm{Xu}$ by doing detailed estimates on the function $\widehat{\beta}(t)$ using the representation (6).

Let $k$ denote the constant time step, $t_{n}=k n$ the $n$th time level, and $\mathbf{U}^{n}$ the approximation of $\mathbf{u}\left(t_{n}\right)$. The backward Euler method is used with $\bar{\partial} \mathbf{U}^{n}=\frac{\mathbf{U}^{n}-\mathbf{U}^{n-1}}{k}$ approximating the derivative $\mathbf{u}^{\prime}$ in (2) at the $n$th time level. For the integral we apply the first-order convolution quadrature introduced by Lubich [7]:

$$
q_{n}(\varphi)=\sum_{j=1}^{n} \beta_{n-j}(k) \varphi^{j},
$$

where $\varphi^{j}=\varphi\left(t_{j}\right)$ and the quadrature weights $\beta_{n-j}(k)$ are the coefficients of the power series

$$
\widehat{\beta}\left(\frac{1-z}{k}\right)=\sum_{j=0}^{\infty} \beta_{j}(k) z^{j} .
$$

This leads to the time discrete problem

$$
\bar{\partial} \mathbf{U}^{n}+q_{n}(\mathbf{A U})=0, \quad \mathbf{U}^{0}=\mathbf{u}_{0} .
$$

Our first theorem generalizes Theorem 1 of [21] by replacing the completely monotonic assumption (4) with (5). 
Theorem 1. If (3) and (5) hold, then

$$
k \sum_{n=1}^{\infty}\left\|\mathbf{U}^{n}\right\| \leq C\left\|\mathbf{A} \mathbf{u}_{0}\right\| .
$$

In order to state our next theorem we must first define some auxiliary functions. For $\sigma+i \tau \notin(-\infty, 0]$, set $\beta(t)=c(t)+\beta(\infty)$, and then let

$$
\begin{aligned}
& \phi(\sigma, \tau)=\int_{0}^{\infty} \cos (\tau t) e^{-\sigma t} \beta(t) d t \quad \text { and } \quad \theta(\sigma, \tau)=\frac{1}{\tau} \int_{0}^{\infty} \sin (\tau t) e^{-\sigma t} \beta(t) d t, \\
& \phi_{c}(\sigma, \tau)=\int_{0}^{\infty} \cos (\tau t) e^{-\sigma t} c(t) d t \quad \text { and } \quad \theta_{c}(\sigma, \tau)=\frac{1}{\tau} \int_{0}^{\infty} \sin (\tau t) e^{-\sigma t} c(t) d t,
\end{aligned}
$$

and for $0<\tau<\infty$, set

$$
\phi_{c}(\tau)=\lim _{\sigma \rightarrow 0+} \phi_{c}(\sigma, \tau)=\int_{0}^{\infty} \cos (\tau t) c(t) d t
$$

and

$$
\theta_{c}(\tau)=\lim _{\sigma \rightarrow 0+} \theta_{c}(\sigma, \tau)=\frac{1}{\tau} \int_{0}^{\infty} \sin (\tau t) c(t) d t
$$

So, for $\sigma+i \tau \notin(-\infty, 0]$, we have

$$
\phi(\sigma, \tau)=\phi_{c}(\sigma, \tau)+\frac{\sigma \beta(\infty)}{\sigma^{2}+\tau^{2}} \quad \text { and } \quad \theta(\sigma, \tau)=\theta_{c}(\sigma, \tau)+\frac{\beta(\infty)}{\sigma^{2}+\tau^{2}},
$$

and then, for $0<\tau<\infty$, we may set

$$
\phi(\tau)=\lim _{\sigma \rightarrow 0+} \phi(\sigma, \tau)=\phi_{c}(\tau) \quad \text { and } \quad \theta(\tau)=\lim _{\sigma \rightarrow 0+} \theta(\sigma, \tau)=\theta_{c}(\tau)+\frac{\beta(\infty)}{\tau^{2}} .
$$

We see then that the Fourier transform of $\beta(t)$,

$$
\widetilde{\beta}(\tau)=\int_{0}^{\infty} e^{-i \tau t} \beta(t) d t
$$

obeys the relation

$$
\widetilde{\beta}(\tau)=\phi(\tau)-i \tau \theta(\tau),
$$

and further, the Laplace transform obeys

$$
\widehat{\beta}(\sigma+i \tau)=\phi(\sigma, \tau)-i \tau \theta(\sigma, \tau) .
$$

As a consequence of Theorem 2.2 and Corollary 2.1 of Carr and Hannsgen [1], (3) and (5) imply

$$
\limsup _{\tau \rightarrow \infty} \frac{\theta_{c}(\tau)}{\phi_{c}(\tau)}<\infty
$$

By (4.3) of [1], we see that $\tau^{-2}=o\left(\theta_{c}(\tau)\right)(\tau \rightarrow \infty)$, so it follows that (3) and (5) imply that

$$
\limsup _{\tau \rightarrow \infty} \frac{\theta(\tau)}{\phi(\tau)}<\infty
$$

Copyright $\odot$ by SIAM. Unauthorized reproduction of this article is prohibited. 
If instead our kernel $\beta(t)$ is such that

$$
\limsup _{\tau \rightarrow \infty} \frac{\tau^{\frac{1}{3}} \theta(\tau)}{\phi(\tau)}<\infty
$$

holds, then we can obtain the following theorem which generalizes Theorem 2 of [21].

Theorem 2. If (3), (5), and (18) hold, then

$$
k \sum_{n=1}^{\infty}\left\|\mathbf{U}^{n}\right\| \leq C\left\|\mathbf{u}_{0}\right\| .
$$

We note that (18) is a significantly weaker frequency condition upon $\beta(t)$ than is employed in Theorem 2 of [21], namely, that

$$
\limsup _{\tau \rightarrow \infty} \frac{\tau \theta(\tau)}{\phi(\tau)}<\infty .
$$

For example, if $\beta(t)$ satisfies (5) and behaves like $(-\log (t))^{p}(p>0)$ near the origin, then an easy calculation utilizing the relations (37) and (39) shows that (18) is satisfied, but not (20). We see that in Theorem 1 we are allowed a wider class of kernel functions $\beta(t)$, but we have the more restrictive requirement that $\mathbf{u}_{0} \in \mathcal{D}(\mathbf{A})$, whereas Theorem 2 places greater restrictions upon $\beta(t)$, yet allows $\mathbf{u}_{0}$ to be any element of $\mathbf{H}$.

The resolvent kernel of (2) is defined by the formula

$$
\mathbf{U}(t)=\int_{-\infty}^{\infty} u(t, \lambda) d \mathbf{E}_{\lambda},
$$

where $u(t, \lambda)$ is the solution of the scalar Volterra integrodifferential equation

$$
u^{\prime}(t)+\lambda \int_{0}^{t} \beta(t-s) u(s) d s=0, \quad u(0)=1 ;
$$

the parameter $\lambda$ satisfies $\lambda_{0} \leq \lambda$ and $0 \leq t$.

It is clear from (21) that

$$
\sup _{\lambda_{0} \leq \lambda}|u(t, \lambda)| \rightarrow 0, \quad t \rightarrow \infty
$$

and

$$
\int_{0}^{\infty} \sup _{\lambda_{0} \leq \lambda}|u(t, \lambda)| d t<\infty
$$

imply, respectively,

$$
\|\mathbf{U}(t)\| \rightarrow 0, \quad t \rightarrow \infty
$$

and

$$
\int_{0}^{\infty}\|\mathbf{U}(t)\| d t<\infty
$$

Then the resolvent formula

$$
\mathbf{y}(t)=\mathbf{U}(t) \mathbf{y}_{0}+\int_{0}^{t} \mathbf{U}(t-s) \mathbf{f}(s) d s
$$

Copyright $@$ by SIAM. Unauthorized reproduction of this article is prohibited. 
can be used to obtain precise asymptotic information $(t \rightarrow \infty)$ about the solution $\mathbf{y}(t)$ of the initial value problem

$$
\mathbf{u}^{\prime}(t)+\int_{0}^{t} \beta(t-s) \mathbf{A u}(s) d s=\mathbf{f}(t), \quad t \geq 0, \quad \mathbf{u}(0)=\mathbf{u}_{0} .
$$

In [1] several sufficient conditions are given on $\beta(t)$ such that (23) and (24) hold. One easily stated consequence of [1] which is relevant here is that (23) and (24) both hold, and, as a consequence, (25) and (26) when $\beta(t)$ satisfies (5).

In [21] the stability of a numerical scheme for approximating the solution of (2) is a discrete analogue of (26). Let $\left\{U^{n}(\lambda)\right\}_{n=0}^{\infty}$ be a real sequence satisfying the difference equation

$$
\frac{U^{n}(\lambda)-U^{n-1}(\lambda)}{k}+\lambda q_{n}(U(\lambda))=0, \quad n \geq 1, \quad U^{0}(\lambda)=1 .
$$

It follows from the functional calculus for spectral decompositions (see [17]) that the solution to (11) may be representated as

$$
\mathbf{U}^{n}=\int_{-\infty}^{\infty} U^{n}(\lambda) d \mathbf{E}_{\lambda} \mathbf{u}_{0} .
$$

We note that Lemma 1 from [6] implies that $e^{-\sigma t} \beta(t)$ and $\left(e^{-\sigma t} \beta(t)\right)^{\prime}$ are convex for $\sigma>0$. Also, from Theorem 2 and the comments following it in [13] we find that $\beta(t)$ is positive-definite, implying that $\operatorname{Re}(\widehat{\beta}(s))>0$ whenever $s=\sigma+i \tau$ with $\sigma>0$. Then, by an argument similar to that in Lemma 3.1 of [8], we find that the quadrature (9) is positive-definite in the sense that for each function $\varphi:(0, \infty) \rightarrow \mathbf{H}$ and each positive integer $N$, we have

$$
Q_{N}(\varphi) \equiv k \sum_{n=1}^{N}\left(q_{n}(\varphi), \varphi^{n}\right) \geq 0
$$

To see this, set

$\widetilde{\varphi}(t)=\sum_{j=1}^{N} \varphi^{j} t^{j}, \quad \widetilde{\beta}(t)=\sum_{j=0}^{\infty} \beta_{j}(k) t^{j} \quad$ and $\quad Q_{N, r}(\varphi)=k \sum_{n=1}^{N} \sum_{j=1}^{n} \beta_{n-j}(k) r^{n-j}\left(\varphi^{j}, \varphi^{n}\right)$

for $0<r<1$. Then, it is straightforward to show that

$$
Q_{N, r}(\varphi)=\frac{k}{2 \pi} \int_{0}^{2 \pi} \widetilde{\beta}\left(r e^{i \theta}\right)\left\|\widetilde{\varphi}\left(e^{i \theta}\right)\right\|^{2} d \theta .
$$

As $\mathbf{H}$ is a real Hilbert space, it follows from (10) that $Q_{N, r}(\varphi) \geq 0$. Then, by (9) we find that $Q_{N, r}(\varphi) \rightarrow Q_{N}(\varphi) \quad(r \uparrow 1)$, from which (31) follows.

By an argument very similar to that given in Lemma 3.1 of [10], it can be shown that (31) implies that

$$
\left\|\mathbf{U}^{n}\right\| \leq\left\|\mathbf{u}_{0}\right\|
$$

Then (32) implies that

$$
k \sum_{n=1}^{m}\left\|\mathbf{U}^{n}\right\| \leq t_{m}\left\|\mathbf{u}_{0}\right\|
$$

Copyright (C) by SIAM. Unauthorized reproduction of this article is prohibited. 
Thus, by (30) and (33), we see that it is sufficient to show that

$$
k \sum_{n=m+1}^{\infty} \sup _{\lambda \geq \lambda_{0}}\left|U^{n}(\lambda) \lambda^{-1}\right| \leq C
$$

and

$$
k \sum_{n=m+1}^{\infty} \sup _{\lambda \geq \lambda_{0}}\left|U^{n}(\lambda)\right| \leq C
$$

to prove Theorems 1 and 2 , respectively.

Equations (2) and (28) arise in the theory of linear viscoelasticity. A nice survey may be found in [16]. For a comprehensive treatment of Volterra equations see [5] or [15]. Another interesting work on the numerical approximation of the solution of (2) which assumes (3) and (5) is given by Xu in [24, Remark 2.3] in which a Galerkin method is studied. For a numerical solution utilizing quadrature applied to the inverse Laplace transform form of the solution, see [11]. For a second-order accurate finite difference solution, see [9]. A solution utilizing finite difference convolution quadrature is given in [3]. For a time-stepping discontinuous Galerkin solution, see [12].

In the next section we establish some preliminary results and in section 3 we present the proofs of our theorems. In all that follows we assume that $\varepsilon>0$ is a sufficiently small fixed constant independent of $k$ whose value will be specified later. We also note that $\mathrm{C}$ is a generic constant whose value may change at each appearance and which depends only upon $\varepsilon$ and $\lambda_{0}$.

2. Preliminary estimates. We begin with a lemma from [21, p. 139], which derives from a lemma in $[1$, p. 967].

Lemma 2.1. If $\beta(t)$ satisfies (3) and (5), then

$$
\begin{gathered}
\frac{1}{2 \sqrt{2}} \int_{0}^{\frac{1}{\tau}} \beta(t) d t \leq|\widetilde{\beta}(\tau)| \leq 4 \int_{0}^{\frac{1}{\tau}} \beta(t) d t, \quad \tau>0, \\
\frac{1}{5} \int_{0}^{\frac{1}{\tau}} t \beta(t) d t \leq \theta(\tau) \leq 12 \int_{0}^{\frac{1}{\tau}} t \beta(t) d t, \quad \tau>0, \\
\left|\widetilde{\beta}^{\prime}(\tau)\right| \leq 40 \int_{0}^{\frac{1}{\tau}} t \beta(t) d t, \quad \tau>0 .
\end{gathered}
$$

Here, recall that $\widetilde{\beta}(\tau)$ is the Fourier transform of $\beta(t)$. We note that these results hold without the convexity of $-\beta^{\prime}(t)$ assumed. As we know that $e^{-\sigma t} \beta(t)$ and $\left(e^{-\sigma t} \beta(t)\right)^{\prime}$ are convex for $\sigma>0$, then with only slight modifications to the proof we obtain results similar to those in Noren (see [14, eq. (4.14)]):

$$
\frac{1}{C} \int_{0}^{\frac{1}{\tau}}-t \beta^{\prime}(t) d t \leq \phi(\tau) \leq C \int_{0}^{\frac{1}{\tau}}-t \beta^{\prime}(t) d t, \quad \tau>0,
$$

and

$$
\frac{1}{C} \int_{0}^{\frac{1}{\tau}}-t\left(e^{-\sigma t} \beta(t)\right)^{\prime} d t \leq \phi(\sigma, \tau) \leq C \int_{0}^{\frac{1}{\tau}}-t\left(e^{-\sigma t} \beta(t)\right)^{\prime} d t, \quad \sigma, \tau>0 .
$$

One consequence of (39) and (40) in the case where $0<\sigma \leq \varepsilon \tau$ is that

$$
\phi(\sigma, \tau) \geq C \int_{0}^{\frac{1}{\tau}}-t\left(e^{-\sigma t} \beta(t)\right)^{\prime} d t \geq C e^{-\frac{\sigma}{\tau}} \int_{0}^{\frac{1}{\tau}}-t \beta^{\prime}(t) d t \geq C \phi(\tau) .
$$


As $e^{-\sigma t} \beta(t)$ satisfies the hypotheses of Lemma 2.1, we obtain the following variants of (36) and (38):

$$
\frac{1}{2 \sqrt{2}} \int_{0}^{\frac{1}{\tau}} e^{-\sigma t} \beta(t) d t \leq\left|\left(e^{-\sigma t} \beta(t)\right)^{\sim}(\tau)\right| \leq 4 \int_{0}^{\frac{1}{\tau}} e^{-\sigma t} \beta(t) d t, \quad \sigma, \tau>0,
$$

and

$$
\left|\frac{d}{d \tau}\left(e^{-\sigma t} \beta(t)\right)^{\sim}(\tau)\right| \leq 40 \int_{0}^{\frac{1}{\tau}} t e^{-\sigma t} \beta(t) d t, \quad \sigma, \tau>0 .
$$

Defining the functions $A(x)=\int_{0}^{x} \beta(t) d t$ and $A_{1}(x)=\int_{0}^{x} t \beta(t) d t$, we also recall a result from Shea and Wainger [19, eq. (1.21)]):

$$
\int_{0}^{\varepsilon} \frac{A_{1}\left(\tau^{-1}\right)}{A^{2}\left(\tau^{-1}\right)} d \tau<\infty
$$

Define the notations

$\sigma=\sigma(k, \nu)=\frac{1-\cos (k \nu)}{k}, \tau=\tau(k, \nu)=\frac{\sin (k \nu)}{k}, s=s(k, \nu)=\sigma+i \tau=\frac{1-e^{-i k \nu}}{k}$,

and

$$
D(s, \lambda)=D(\sigma+i \tau)=\frac{s}{\lambda}+\widehat{\beta}(s)=\frac{\sigma+i \tau}{\lambda}+\widehat{\beta}(\sigma+i \tau) .
$$

Following [1] and [21], we wish to define a strictly increasing function $\omega:\left[\lambda_{0}, \infty\right) \rightarrow$ $[\varepsilon, \infty)$ with $\omega(\lambda) \rightarrow \infty(\lambda \rightarrow \infty)$ and such that $\theta(\omega(\lambda))=\frac{1}{\lambda}$ for $\lambda \geq \lambda_{1} \geq \lambda_{0}$ and, if necessary, $\omega(\lambda)=\varepsilon$ for $\lambda_{1}>\lambda \geq \lambda_{0}$. We note that $\omega$ was continuous in [1], owing to the choice of $\rho=\frac{6}{t_{1}}$ in that paper, and in [21] by the analytic nature of a completely monotonic function. We do not require that $\omega$ be continuous. In this case, slight modification to the proof given in [1, Lemma 5.2] and [2, Lemma 8.1] gives us the following lemma.

Lemma 2.2. If $\beta(t)$ satisfies (3) and (5), then

$$
|D(i \tau, \lambda)| \geq \begin{cases}C_{1} \frac{|\tau-\omega|}{\lambda} & \left(\tau \geq \frac{\omega}{2}\right), \\ C_{1}\left(\tau \int_{0}^{\frac{1}{\tau}} t \beta(t) d t+\int_{0}^{\frac{1}{\tau}} \beta(t) d t\right) & \left(\tau \in\left[\frac{\varepsilon}{2}, \frac{\omega}{2}\right]\right) .\end{cases}
$$

This result also holds if $-\beta^{\prime}(t)$ convex is dropped. We also note that [1] gives us

$$
\omega(\lambda) \leq C \lambda,
$$

and it follows from (6.8) of [1] that, for $\tau \geq \frac{\omega}{2}$, we have

$$
\theta(\tau) \leq C \lambda^{-1} .
$$

We now wish to establish a generalization of (2.9) from [21].

Lemma 2.3. If $\beta(t)$ satisfies (3) and (5) and $0<\sigma \leq \varepsilon \tau<\tau$, then

$$
|\theta(\sigma, \tau)-\theta(\tau)| \leq 29 \varepsilon \theta(\tau) .
$$

Proof. Beginning with the formulas

$$
\theta(\sigma, \tau)=\theta_{c}(\sigma, \tau)+\frac{\beta(\infty)}{\sigma^{2}+\tau^{2}} \quad \text { and } \quad \theta(\tau)=\theta_{c}(\tau)+\frac{\beta(\infty)}{\tau^{2}}
$$

Copyright $@$ by SIAM. Unauthorized reproduction of this article is prohibited. 
we see that

$$
\begin{aligned}
|\theta(\sigma, \tau)-\theta(\tau)| & \leq\left|\theta_{c}(\sigma, \tau)-\theta_{c}(\tau)\right|+\beta(\infty)\left|\frac{1}{\tau^{2}}-\frac{1}{\sigma^{2}+\tau^{2}}\right| \\
& \leq\left|\theta_{c}(\sigma, \tau)-\theta_{c}(\tau)\right|+\beta(\infty) \frac{\varepsilon}{\tau^{2}},
\end{aligned}
$$

so it suffices for us to show that $\left|\theta_{c}(\sigma, \tau)-\theta_{c}(\tau)\right| \leq 29 \varepsilon \theta_{c}(\tau)$. Integrating by parts twice, we get

$$
\begin{aligned}
\theta_{c}(\sigma, \tau)=\frac{1}{\sigma^{2}+\tau^{2}} \int_{0}^{\infty}\left\{\left(1-e^{-\sigma t} \cos (\tau t)\right)-\frac{\sigma}{\tau} e^{-\sigma t} \sin (\tau t)\right\}\left(-c^{\prime}(t)\right) d t \\
=\frac{1}{\left(\sigma^{2}+\tau^{2}\right)^{2}} \int_{0}^{\infty}\left\{\left(\left(\sigma^{2}+\tau^{2}\right) t+\left(\sigma^{2}-\tau^{2}\right) e^{-\sigma t} \frac{\sin (\tau t)}{\tau}\right)\right. \\
\left.-2 \sigma\left(1-e^{-\sigma t} \cos (\tau t)\right)\right\} c^{\prime \prime}(t) d t
\end{aligned}
$$

and

$$
\begin{aligned}
\theta_{c}(\tau) & =\frac{1}{\tau^{2}} \int_{0}^{\infty}(1-\cos (\tau t))\left(-c^{\prime}(t)\right) d t \\
& =\frac{1}{\tau^{2}} \int_{0}^{\infty}\left(t-\frac{\sin (\tau t)}{\tau}\right) c^{\prime \prime}(t) d t
\end{aligned}
$$

The boundary terms vanish due to the relations $t c(t)=t^{2} c^{\prime}(t)=o(1)(t \rightarrow 0+)$ and $t c^{\prime}(t)=o(1)(t \rightarrow \infty)$ from [1]. Then, setting

$$
f(t)=\frac{1}{\left(\sigma^{2}+\tau^{2}\right)^{2}}\left\{\left(\left(\sigma^{2}+\tau^{2}\right) t+\left(\sigma^{2}-\tau^{2}\right) e^{-\sigma t} \frac{\sin (\tau t)}{\tau}\right)-2 \sigma\left(1-e^{-\sigma t} \cos (\tau t)\right)\right\}
$$

and

$$
g(t)=\frac{1}{\tau^{2}}\left(t-\frac{\sin (\tau t)}{\tau}\right)
$$

we see that we need only show that

$$
(1-29 \varepsilon) g(t) \leq f(t) \leq(1+29 \varepsilon) g(t)
$$

to have our result. Since $f^{\prime}(0)=f(0)=g^{\prime}(0)=g(0)=0$ and $(1-\varepsilon) g^{\prime \prime}(t) \leq$ $f^{\prime \prime}(t) \leq g^{\prime \prime}(t)$ for $t \in\left[0, \frac{1}{\tau}\right]$, we may integrate twice over $[0, t]$ for $t \in\left[0, \frac{1}{\tau}\right]$ to obtain $(1-\varepsilon) g(t) \leq f(t) \leq g(t)$ for $t \in\left[0, \frac{1}{\tau}\right]$. First we show that

$$
(1-29 \varepsilon) g(t) \leq f(t) \quad\left(t>\frac{1}{\tau}\right)
$$

Note first that as $0<\sigma \leq \varepsilon \tau<\tau$ and $t>\frac{1}{\tau}$, we have

$\frac{-2 \sigma}{\left(\sigma^{2}+\tau^{2}\right)^{2}}\left(1-e^{-\sigma t} \cos (\tau t)\right) \geq \frac{-2 \sigma}{\tau^{4}}\left(1-e^{-\sigma t} \cos (\tau t)\right) \geq \frac{-4 \varepsilon}{\tau^{3}} \geq \frac{-26 \varepsilon}{\tau^{2}}\left(t-\frac{\sin (\tau t)}{\tau}\right)$.

So, we must show that

$$
\frac{1}{\left(\sigma^{2}+\tau^{2}\right)^{2}}\left(\left(\sigma^{2}+\tau^{2}\right) t+\left(\sigma^{2}-\tau^{2}\right) e^{-\sigma t} \frac{\sin (\tau t)}{\tau}\right) \geq(1-3 \varepsilon)\left(\frac{1}{\tau^{2}}\left(t-\frac{\sin (\tau t)}{\tau}\right)\right) .
$$

Copyright $@$ by SIAM. Unauthorized reproduction of this article is prohibited. 
As $(1+\varepsilon)(1-3 \varepsilon) \leq(1-2 \varepsilon)$ and

$$
\begin{aligned}
\frac{1}{\left(\sigma^{2}+\tau^{2}\right)^{2}}\left(\left(\sigma^{2}+\tau^{2}\right) t+\left(\sigma^{2}-\tau^{2}\right) e^{-\sigma t}\right. & \left.\frac{\sin (\tau t)}{\tau}\right) \\
& \geq \frac{1}{(1+\varepsilon) \tau^{2}}\left(t-\frac{\tau^{2}-\sigma^{2}}{\tau^{2}+\sigma^{2}} e^{-\sigma t} \frac{\sin (\tau t)}{\tau}\right),
\end{aligned}
$$

it suffices to show that (after some rearrangement)

$$
\left(1-\frac{\tau^{2}-\sigma^{2}}{\tau^{2}+\sigma^{2}} e^{-\sigma t}\right) \frac{\sin (\tau t)}{\tau} \geq 2 \varepsilon\left(\frac{\sin (\tau t)}{\tau}-t\right) .
$$

This clearly holds if $\sin (\tau t) \geq 0$, so assume otherwise. Then, as

$$
\begin{aligned}
\left(1-\frac{\tau^{2}-\sigma^{2}}{\tau^{2}+\sigma^{2}} e^{-\sigma t}\right) \frac{\sin (\tau t)}{\tau} & \geq\left(1-\frac{1-\varepsilon}{1+\varepsilon}(1-\varepsilon \tau t)\right) \frac{\sin (\tau t)}{\tau} \\
& =\left(\frac{2 \varepsilon}{1+\varepsilon}+\left(\frac{1-\varepsilon}{1+\varepsilon}\right) \varepsilon \tau t\right) \frac{\sin (\tau t)}{\tau},
\end{aligned}
$$

(50) follows since $2 \varepsilon \leq 2 \varepsilon(1+\varepsilon)$ and $\left(\frac{1-\varepsilon}{1+\varepsilon}\right) \sin (\tau t) \geq-1$. We now show that

$$
f(t) \leq(1+29 \varepsilon) g(t) \quad\left(t>\frac{1}{\tau}\right) .
$$

Note that as $0<\sigma \leq \varepsilon \tau<\tau$ and $t>\frac{1}{\tau}$, we have

$$
f(t) \leq \frac{1}{\sigma^{2}+\tau^{2}}\left(t-\frac{\tau^{2}-\sigma^{2}}{\tau^{2}+\sigma^{2}} e^{-\sigma t} \frac{\sin (\tau t)}{\tau}\right) \leq \frac{1}{\tau^{2}}\left(t-\frac{\tau^{2}-\sigma^{2}}{\tau^{2}+\sigma^{2}} e^{-\sigma t} \frac{\sin (\tau t)}{\tau}\right) .
$$

So, we need only show that (after some rearrangement)

$$
\left(1-\frac{\tau^{2}-\sigma^{2}}{\tau^{2}+\sigma^{2}} e^{-\sigma t}\right) \frac{\sin (\tau t)}{\tau} \leq 29 \varepsilon\left(t-\frac{\sin (\tau t)}{\tau}\right) .
$$

This clearly holds if $\sin (\tau t) \leq 0$, so assume otherwise. Then, we see that

$$
\begin{aligned}
\left(1-\frac{\tau^{2}-\sigma^{2}}{\tau^{2}+\sigma^{2}} e^{-\sigma t}\right) \frac{\sin (\tau t)}{\tau} & \leq\left(1-\frac{1-\varepsilon}{1+\varepsilon}(1-\varepsilon \tau t)\right) \frac{\sin (\tau t)}{\tau} \\
& \leq \varepsilon(2+\tau t) \frac{\sin (\tau t)}{\tau} \\
& \leq 3 \varepsilon t,
\end{aligned}
$$

from which (51) follows, as $\sin (\tau t) \leq \frac{26}{29} \tau t$ for $t>\frac{1}{\tau}$. Then, combining (50) and (51), we obtain (49), which proves the lemma.

We next wish to prove the following estimate.

LEMmA 2.4. If $\beta(t)$ satisfies (3) and (5), $k \leq 1, \frac{\pi-\varepsilon}{k} \leq \nu \leq \frac{\pi}{k}$ and $\varepsilon \leq \frac{1}{5}$, then

$$
\phi(\sigma, \tau) \geq \frac{1}{2} \widehat{\beta}(\sigma) .
$$

Proof. Beginning with the formulas

$\phi(\sigma, \tau)=\int_{0}^{\infty} \cos (\tau t) e^{-\sigma t} c(t) d t+\frac{\sigma \beta(\infty)}{\sigma^{2}+\tau^{2}} \quad$ and $\quad \widehat{\beta}(\sigma)=\int_{0}^{\infty} e^{-\sigma t} c(t) d t+\frac{\beta(\infty)}{\sigma}$,

Copyright $@$ by SIAM. Unauthorized reproduction of this article is prohibited. 
we note that as $\frac{\pi-\varepsilon}{k} \leq \nu \leq \frac{\pi}{k}$ gives us $\frac{2-\varepsilon}{k} \leq \sigma \leq \frac{2}{k}$ and $0 \leq \tau \leq \frac{\varepsilon}{k}$, we find that $\frac{\sigma}{\sigma^{2}+\tau^{2}} \geq \frac{(2-\varepsilon) k}{4+\varepsilon^{2}} \geq \frac{45 k}{101}>\frac{k}{4-2 \varepsilon} \geq \frac{1}{2 \sigma}$, so we need only show that $\phi_{c}(\sigma, \tau) \geq \frac{1}{2} \widehat{c}(\sigma)$. Integrating by parts, we obtain

$$
\phi_{c}(\sigma, \tau)=\frac{1}{\sigma^{2}+\tau^{2}} \int_{0}^{\infty}\left[\sigma\left(1-e^{-\sigma t} \cos (\tau t)\right)+\tau e^{-\sigma t} \sin (\tau t)\right]\left(-c^{\prime}(t)\right) d t
$$

and

$$
\widehat{c}(\sigma)=\frac{1}{\sigma} \int_{0}^{\infty}\left(1-e^{-\sigma t}\right)\left(-c^{\prime}(t)\right) d t .
$$

The boundary terms vanish as in the proof of Lemma 2.3. Then, setting

$$
f(t)=\frac{1}{\sigma^{2}+\tau^{2}}\left[\sigma\left(1-e^{-\sigma t} \cos (\tau t)\right)+\tau e^{-\sigma t} \sin (\tau t)\right] \quad \text { and } \quad g(t)=\frac{1}{\sigma}\left(1-e^{-\sigma t}\right),
$$

we see that for $t \leq \frac{1}{\tau}$, we have $f^{\prime}(t)=e^{-\sigma t} \cos (\tau t) \geq \frac{1}{2} e^{-\sigma t}=\frac{1}{2} g^{\prime}(t)$, so as $f(0)=$ $g(0)=0$, we may integrate over $[0, t]$, for $t \leq \frac{1}{\tau}$, to obtain $f(t) \geq \frac{1}{2} g(t)$ for $t \leq \frac{1}{\tau}$. Thus, it is only a matter of showing that $f(t) \geq \frac{1}{2} g(t)$ for $t>\frac{1}{\tau}$ to establish (52). As $\frac{2-\varepsilon}{k} \leq \sigma \leq \frac{2}{k}$ and $0 \leq \tau \leq \frac{\varepsilon}{k}$, it follows that for $\varepsilon \leq \frac{1}{5}$ we have $\frac{\sigma}{\tau} \geq \frac{2-\varepsilon}{\varepsilon} \geq 9$; so clearly we have $e^{\frac{-\sigma}{\tau}} \leq\left(1-e^{-\sigma t}\right)$ for $t>\frac{1}{\tau}$. Thus, for $t>\frac{1}{\tau}$, we see that

$$
\begin{aligned}
f(t) & \geq \frac{k^{2}}{4+\varepsilon^{2}}\left(\frac{2-\varepsilon}{k}\left(1-e^{-\sigma t}\right)-\frac{\varepsilon}{k} e^{\frac{-\sigma}{\tau}}\right) \\
& \geq \frac{k}{4+\varepsilon}\left((2-\varepsilon)\left(1-e^{-\sigma t}\right)-\varepsilon\left(1-e^{-\sigma t}\right)\right) \\
& =k\left(\frac{2-2 \varepsilon}{4+\varepsilon}\right)\left(1-e^{-\sigma t}\right) \\
& \geq \frac{8 k}{21}\left(1-e^{-\sigma t}\right) \\
& \geq \frac{1}{2} g(t),
\end{aligned}
$$

which proves the lemma.

We next wish to extend Lemma 3.1 in [21].

Lemma 2.5. If $\beta(t)$ satisfies (3) and (5), $\lambda \geq \lambda_{0}$, and $k<1$, then

$$
k \sum_{n=1}^{\infty}\left|U^{n}(\lambda)\right| \leq C \lambda
$$

Proof. Following [21], we define the generating function of $\left\{U^{n}(\lambda)\right\}_{n=0}^{\infty}$ to be

$$
\widetilde{U}(z, \lambda)=\sum_{n=1}^{\infty} U^{n}(\lambda) z^{n}
$$

which may be shown to obey the relations

$$
\widetilde{U}(z, \lambda)=\frac{z}{k} \frac{1}{\left(\frac{1-z}{k}\right)+\lambda \widehat{\beta}\left(\frac{1-z}{k}\right)}=\frac{z}{k} \widehat{u}\left(\frac{1-z}{k}, \lambda\right) .
$$

Copyright $@$ by SIAM. Unauthorized reproduction of this article is prohibited. 
Then, an application of Hardy's inequality [4, p. 48] gives us

$$
\begin{aligned}
\sum_{n=1}^{\infty}\left|U^{n}(\lambda)\right| & \leq 2 \sum_{n=1}^{\infty} \frac{n\left|U^{n}(\lambda)\right|}{n+1} \leq \int_{-\pi}^{\pi}\left|\widetilde{U}^{\prime}\left(e^{i \nu}, \lambda\right)\right| d \nu \\
& \leq 2 k \int_{0}^{\frac{\pi}{k}}\left|\widetilde{U}^{\prime}\left(e^{-i k \nu}, \lambda\right)\right| d \nu=2 k\left\{\int_{0}^{\varepsilon}+\int_{\varepsilon}^{\frac{\varepsilon}{k}}+\int_{\frac{\varepsilon}{k}}^{\frac{\pi}{k}}\right\}\left|\widetilde{U}^{\prime}\left(e^{-i k \nu}, \lambda\right)\right| d \nu,
\end{aligned}
$$

where

$$
\widetilde{U}^{\prime}(z, \lambda)=\frac{1}{k} \frac{1}{\left[\left(\frac{1-z}{k}\right)+\lambda \widehat{\beta}\left(\frac{1-z}{k}\right)\right]^{2}}\left[\frac{1}{k}+\lambda \widehat{\beta}\left(\frac{1-z}{k}\right)+\frac{\lambda z}{k} \widehat{\beta}^{\prime}\left(\frac{1-z}{k}\right)\right] .
$$

Thus, our extension reduces to establishing the three estimates

$$
\begin{aligned}
& k^{2} \int_{0}^{\varepsilon}\left|\widetilde{U}^{\prime}\left(e^{-i k \nu}, \lambda\right)\right| d \nu \leq C \lambda^{-1}, \\
& k^{2} \int_{\varepsilon}^{\frac{\varepsilon}{k}}\left|\widetilde{U}^{\prime}\left(e^{-i k \nu}, \lambda\right)\right| d \nu \leq C \lambda, \\
& k^{2} \int_{\frac{\varepsilon}{k}}^{\frac{\pi}{k}}\left|\widetilde{U}^{\prime}\left(e^{-i k \nu}, \lambda\right)\right| d \nu \leq C k \lambda .
\end{aligned}
$$

We prove (55) first. For $\varepsilon, k<1$, we see that when $0 \leq \nu \leq \varepsilon$, we get

$$
\frac{\nu}{2} \leq \tau(k, \nu) \leq \nu, \quad \sigma(k, \nu) \leq \varepsilon \tau \leq \tau, \quad \cos (k \nu) \geq \frac{1}{2}, \quad \frac{\sin (k \nu)}{k} \leq \varepsilon .
$$

By (40) and (58), we see that

$$
\operatorname{Re} \widehat{\beta}(\sigma+i \tau) \geq C \int_{0}^{\frac{1}{\tau}}-t e^{-\sigma t} \beta^{\prime}(t) d t \geq C \int_{0}^{\frac{1}{\varepsilon}}-t \beta^{\prime}(t) d t \geq \sqrt{2} C \frac{\tau}{\lambda} .
$$

We may obviously assume $C<1$ in (59), giving

$$
\begin{aligned}
|D(\sigma+i \tau, \lambda)|^{2} & =\left|\phi(\sigma, \tau)+\frac{\sigma}{\lambda}\right|^{2}+\left|\tau \theta(\sigma, \tau)-\frac{\tau}{\lambda}\right|^{2} \\
& \geq \phi^{2}(\sigma, \tau)+C^{2}\left|\tau \theta(\sigma, \tau)-\frac{\tau}{\lambda}\right|^{2} \\
& \geq \frac{\phi^{2}(\sigma, \tau)}{2}+C^{2} \frac{\tau^{2}}{\lambda^{2}}+C^{2}\left|\tau \theta(\sigma, \tau)-\frac{\tau}{\lambda}\right|^{2} \\
& =\frac{\phi^{2}(\sigma, \tau)}{2}+C^{2} \frac{\tau^{2}}{2}\left(\theta(\sigma, \tau)-\frac{2}{\lambda}\right)^{2}+C^{2} \frac{\tau^{2}}{2} \theta^{2}(\sigma, \tau) \\
& \geq C^{2}\left(\frac{\phi^{2}(\sigma, \tau)+\tau^{2} \theta^{2}(\sigma, \tau)}{2}+\frac{\tau^{2}}{2}\left(\theta(\sigma, \tau)-\frac{2}{\lambda}\right)^{2}\right) \\
& \geq C^{2}\left(\frac{\phi^{2}(\sigma, \tau)+\tau^{2} \theta^{2}(\sigma, \tau)}{2}\right)
\end{aligned}
$$

so we obtain

$$
|D(\sigma+i \tau, \lambda)| \geq C|\widehat{\beta}(\sigma+i \tau)|
$$

Copyright (c) by SIAM. Unauthorized reproduction of this article is prohibited. 
Then, (36), (42), and (58) give us

$$
|\widehat{\beta}(\sigma+i \tau)| \geq C \int_{0}^{\frac{1}{\tau}} e^{-\sigma t} \beta(t) d t \geq C|\widetilde{\beta}(\tau)| \geq C \int_{0}^{\frac{1}{\varepsilon}} \beta(t) d t \geq C|\widetilde{\beta}(\varepsilon)|,
$$

so (60) implies that

$$
|D(\sigma+i \tau, \lambda)| \geq C|\widetilde{\beta}(\tau)| \geq C|\widetilde{\beta}(\varepsilon)| .
$$

Note that (36) and (42) give us

$$
|\widehat{\beta}(\sigma+i \tau)|=\left|\left(e^{-\sigma t} \beta(t)\right)^{\sim}(\tau)\right| \leq C|\widetilde{\beta}(\tau)|, \quad \tau>0 .
$$

We also see that (37) and (43) imply

$$
\left|\widehat{\beta}^{\prime}(\sigma+i \tau)\right|=\left|\frac{d}{d \tau}\left(e^{-\sigma t} \beta(t)\right)^{\sim}(\tau)\right| \leq C \theta(\tau), \quad \sigma, \tau>0 .
$$

Then it follows from (36), (37), (44), (61), (62), and (63) that

$$
\begin{aligned}
k^{2} \int_{0}^{\varepsilon}\left|\widetilde{U}^{\prime}\left(e^{-i k \nu}, \lambda\right)\right| d \nu & \leq \int_{0}^{\varepsilon} \frac{1}{\lambda^{2}\left|D^{2}(\sigma+i \tau, \lambda)\right|}\left[1+\lambda k|\widehat{\beta}(\sigma+i \tau)|+\lambda\left|\widehat{\beta}^{\prime}(\sigma+i \tau)\right|\right] d \nu \\
& \leq \frac{C}{\lambda} \int_{0}^{\varepsilon} \frac{1}{|\widetilde{\beta}(\varepsilon)|^{2}}+\frac{k|\widetilde{\beta}(\tau)|}{|\widetilde{\beta}(\tau)| \widetilde{\beta}(\varepsilon) \mid}+\frac{\theta(\tau)}{|\widetilde{\beta}(\tau)|^{2}} d \tau \\
& \leq C \lambda^{-1}
\end{aligned}
$$

so estimate (55) holds. We now show (56). Here $\varepsilon \leq \nu \leq \frac{\varepsilon}{k}$ and for $\varepsilon, k \leq 1$ we have

$$
\frac{\nu}{2} \leq \tau(k, \nu) \leq \nu, \quad \sigma(k, \nu) \leq \varepsilon \tau(k, \nu) \leq \tau(k, \nu), \quad \cos (k \nu) \geq \frac{1}{2} .
$$

We shall establish the following estimates on $|D(\sigma+i \tau, \lambda)|$ when $\varepsilon \leq \min \left\{\frac{C_{1}}{1392}, \frac{5}{348}\right\}$ :

$$
|D(\sigma+i \tau, \lambda)| \geq C\left(\tau \theta(\tau)+\int_{0}^{\frac{1}{\tau}} \beta(t) d t\right), \quad \tau \in\left[\frac{\varepsilon}{2}, \frac{\omega}{2}\right] \bigcap\left[\frac{\varepsilon}{2}, \frac{\varepsilon}{k}\right],
$$

and

$$
|D(\sigma+i \tau, \lambda)| \geq C \frac{\tau-\omega}{\lambda}, \quad \tau \geq 2 \omega .
$$

We show (65) first. To establish

$$
|D(\sigma+i \tau, \lambda)| \geq C \tau \theta(\tau), \quad \tau \in\left[\frac{\varepsilon}{2}, \frac{\omega}{2}\right] \bigcap\left[\frac{\varepsilon}{2}, \frac{\varepsilon}{k}\right],
$$

note that in the case where

$$
\tau\left|\theta(\tau)-\frac{1}{\lambda}\right|<\frac{C_{1} \tau}{2} \int_{0}^{\frac{1}{\tau}} t \beta(t) d t
$$

we can use (37), (41), (45), and (64) to show that

$$
\begin{aligned}
|D(\sigma+i \tau, \lambda)| & \geq C \phi(\tau) \geq C\left(C_{1} \tau \int_{0}^{\frac{1}{\tau}} t \beta(t) d t-\tau\left|\theta(\tau)-\frac{1}{\lambda}\right|\right) \\
& \geq C\left(\frac{C_{1} \tau}{2} \int_{0}^{\frac{1}{\tau}} t \beta(t) d t\right) \geq C \tau \theta(\tau) .
\end{aligned}
$$

Copyright $@$ by SIAM. Unauthorized reproduction of this article is prohibited. 
Similarly, when

$$
\tau\left|\theta(\tau)-\frac{1}{\lambda}\right| \geq \frac{C_{1} \tau}{2} \int_{0}^{\frac{1}{\tau}} t \beta(t) d t
$$

we find that (37) and (48) give us

$$
\tau\left|\theta(\tau)-\frac{1}{\lambda}\right| \geq \frac{C_{1} \tau}{2} \int_{0}^{\frac{1}{\tau}} t \beta(t) d t \geq \frac{C_{1} \tau}{24} \theta(\tau) \geq 2 \tau|\theta(\sigma, \tau)-\theta(\tau)| .
$$

Thus, it follows by (37) that

$$
\begin{aligned}
|D(\sigma+i \tau, \lambda)| & \geq \tau\left|\theta(\sigma, \tau)-\frac{1}{\lambda}\right|=\tau\left|\left(\theta(\tau)-\frac{1}{\lambda}\right)+(\theta(\sigma, \tau)-\theta(\tau))\right| \geq \frac{\tau}{2}\left|\theta(\tau)-\frac{1}{\lambda}\right| \\
& \geq \frac{C_{1} \tau}{2} \int_{0}^{\frac{1}{\tau}} t \beta(t) d t \geq C \tau \theta(\tau) .
\end{aligned}
$$

This establishes (67). The estimate

$$
|D(\sigma+i \tau, \lambda)| \geq C \int_{0}^{\frac{1}{\tau}} \beta(t) d t, \quad \tau \in\left[\frac{\varepsilon}{2}, \frac{\omega}{2}\right] \bigcap\left[\frac{\varepsilon}{2}, \frac{\varepsilon}{k}\right],
$$

follows from the same argument with $\tau \int_{0}^{\frac{1}{\tau}} t \beta(t) d t$ replaced by $\int_{0}^{\frac{1}{\tau}} \beta(t) d t$. Then, combining (67) and (68), we have (65). To prove (66), we note that (41) gives us

$$
|D(\sigma+i \tau, \lambda)| \geq C \phi(\tau),
$$

which establishes the estimate when $\phi(\tau) \geq \frac{C_{1}}{2} \frac{\tau-\omega}{\lambda}$. Thus, assume $\phi(\tau)<\frac{C_{1}}{2} \frac{\tau-\omega}{\lambda}$. Then, (45) gives us

$$
C_{1} \frac{\tau-\omega}{\lambda} \leq|D(i \tau, \lambda)| \leq \phi(\tau)+\tau\left|\theta(\tau)-\frac{1}{\lambda}\right| \leq \frac{C_{1}}{2} \frac{\tau-\omega}{\lambda}+\tau\left|\theta(\tau)-\frac{1}{\lambda}\right|,
$$

so

$$
\left|\frac{1}{\lambda}-\theta(\tau)\right| \geq \frac{C_{1}(\tau-\omega)}{2 \tau \lambda}, \quad \tau \geq 2 \omega
$$

As $\theta(\tau)=\theta_{c}(\tau)+\frac{\beta(\infty)}{\tau^{2}}$, we see by (4.4) of [1] that $\theta(\tau)$ is decreasing. It follows by our construction of $\omega(\lambda)$ that in the case where $\lambda \geq \lambda_{1}$, or by (69), in the case where $\lambda_{0} \geq \lambda>\lambda_{1}$ with $\frac{1}{\lambda}-\theta(\tau) \geq \frac{C_{1}(\tau-\omega)}{2 \tau \lambda}$, that as $\varepsilon \leq \min \left\{\frac{C_{1}}{1392}, \frac{5}{348}\right\}$, we have

$$
\begin{aligned}
\theta(\sigma, \tau) & \leq(1+29 \varepsilon) \theta(\tau)=(1+29 \varepsilon) \frac{1}{\lambda}-(1+29 \varepsilon)\left(\frac{1}{\lambda}-\theta(\tau)\right) \\
& \leq \frac{1}{\lambda}-\frac{C_{1}(\tau-\omega)}{4 \tau \lambda}\left(2+58 \varepsilon-\frac{116 \varepsilon \tau}{C_{1}(\tau-\omega)}\right) \\
& \leq \frac{1}{\lambda}-\frac{C_{1}(\tau-\omega)}{4 \tau \lambda}\left(2-\frac{232 \varepsilon}{C_{1}}\right) \\
& \leq \frac{1}{\lambda}-\frac{C_{1}(\tau-\omega)}{4 \tau \lambda}
\end{aligned}
$$

Copyright $@$ by SIAM. Unauthorized reproduction of this article is prohibited. 
so we find that

$$
|D(\sigma+i \tau, \lambda)| \geq \tau\left(\frac{1}{\lambda}-\theta(\sigma, \tau)\right) \geq C \frac{\tau-\omega}{\lambda}
$$

Similarly, by (69), in the case where $\lambda_{0} \geq \lambda>\lambda_{1}$ with $\frac{1}{\lambda}-\theta(\tau)<-\frac{C_{1}(\tau-\omega)}{2 \tau \lambda}$, we see that as $\varepsilon \leq \min \left\{\frac{C_{1}}{1392}, \frac{5}{348}\right\}$ and as $\frac{\tau}{\tau-\omega} \leq 2$ for $\tau \geq 2 \omega$, we have

$$
\begin{aligned}
\theta(\sigma, \tau) & \geq(1-29 \varepsilon) \theta(\tau)=(1-29 \varepsilon) \frac{1}{\lambda}+(1-29 \varepsilon)\left(\theta(\tau)-\frac{1}{\lambda}\right) \\
& \geq \frac{1}{\lambda}+\frac{C_{1}(\tau-\omega)}{4 \tau \lambda}\left(2-58 \varepsilon-\frac{116 \varepsilon \tau}{C_{1}(\tau-\omega)}\right) \\
& \geq \frac{1}{\lambda}+\frac{C_{1}(\tau-\omega)}{4 \tau \lambda}\left(2-58 \varepsilon-\frac{232 \varepsilon}{C_{1}}\right) \\
& \geq \frac{1}{\lambda}+\frac{C_{1}(\tau-\omega)}{4 \tau \lambda}
\end{aligned}
$$

so we obtain

$$
|D(\sigma+i \tau, \lambda)| \geq \tau\left(\theta(\sigma, \tau)-\frac{1}{\lambda}\right) \geq C \frac{\tau-\omega}{\lambda} .
$$

Combining (70) and (71) completes the proof of (66).

Next, let $E_{1}=\left[\frac{\varepsilon}{2}, \frac{\omega}{2}\right] \cap\left[\frac{\varepsilon}{2}, \frac{\varepsilon}{k}\right], E_{2}=\left[\frac{\omega}{2}, 2 \omega\right] \cap\left[\frac{\varepsilon}{2}, \frac{\varepsilon}{k}\right]$, and $E_{3}=[2 \omega, \infty) \cap\left[\frac{\varepsilon}{2}, \frac{\varepsilon}{k}\right]$. Note that our construction of $\omega$ and the decreasing nature of $\theta(\tau)$ give us $\lambda \theta(\tau) \geq C$ for $\tau \in E_{1}$. Also, by (36), we see that $|\widetilde{\beta}(\tau)| \leq C$ for $\tau \in E_{3}$. Then by (17), (36), (41), (48), (62), (63), (65), and (66), we see that

$$
\begin{aligned}
& k^{2} \int_{\varepsilon}^{\frac{\varepsilon}{k}}\left|\widetilde{U}^{\prime}\left(e^{-i k \nu}, \lambda\right)\right| d \nu \\
& \leq C\left\{\int_{E_{1}}+\int_{E_{2}}+\int_{E_{3}}\right\} \frac{1}{\lambda^{2}\left|D^{2}(\sigma+i \tau, \lambda)\right|}\left[1+\lambda k|\widehat{\beta}(\sigma+i \tau)|+\lambda\left|\widehat{\beta}^{\prime}(\sigma+i \tau)\right|\right] d \tau \\
& \leq C\left(\int_{\frac{\varepsilon}{2}}^{\frac{\omega}{2}} \frac{d \tau}{\tau^{2}}+k \int_{E_{1}} \frac{A\left(\tau^{-1}\right)}{\lambda \tau \theta(\tau) A\left(\tau^{-1}\right)} d \tau\right) \\
&+ \frac{C}{\lambda}\left(\int_{E_{2}} \frac{d \tau}{\lambda \phi^{2}(\tau)}+k \frac{\phi(\tau)+\tau \theta(\tau)}{\phi^{2}(\tau)}+\frac{\theta(\tau)}{\phi^{2}(\tau)} d \tau\right) \\
&+ C\left(\int_{E_{3}} \frac{1+\lambda(1+\theta(\tau))}{(\tau-\omega)^{2}} d \tau\right),
\end{aligned}
$$

so by (46) and (47) we find that

$$
k^{2} \int_{\varepsilon}^{\frac{\varepsilon}{k}}\left|\widetilde{U}^{\prime}\left(e^{-i k \nu}, \lambda\right)\right| d \nu \leq C(1+\lambda+k \lambda) \leq C \lambda
$$

which establishes (56). We now establish (57). Here $\frac{\varepsilon}{k} \leq \nu \leq \frac{\pi}{k}$, and we have

$$
\sigma(k, \nu) \geq \frac{1-\cos (\varepsilon)}{k} \equiv \frac{C(\varepsilon)}{k}, \quad|D(\sigma+i \tau)| \geq \frac{\sigma}{\lambda} \geq \frac{C(\varepsilon)}{k \lambda} .
$$

Copyright (c) by SIAM. Unauthorized reproduction of this article is prohibited. 
Then, as $k \leq 1$, we have

$$
|\widehat{\beta}(\sigma+i \tau)|=\left|\int_{0}^{\infty} e^{-(\sigma+i \tau) t} \beta(t) d t\right| \leq \int_{0}^{\infty} e^{-C(\varepsilon) t} \beta(t) d t=\widehat{\beta}(C(\varepsilon))
$$

and

$$
\left|\widehat{\beta}^{\prime}(\sigma+i \tau)\right|=\left|\int_{0}^{\infty}-t e^{-(\sigma+i \tau) t} \beta(t) d t\right| \leq \int_{0}^{\infty} t e^{-C(\varepsilon) t} \beta(t) d t=\left|\widehat{\beta}^{\prime}(C(\varepsilon))\right| .
$$

So, (72), (73), and (74) give us

$$
\begin{aligned}
k^{2} \int_{\frac{\varepsilon}{k}}^{\frac{\pi}{k}}\left|\widetilde{U}^{\prime}\left(e^{-i k \nu}, \lambda\right)\right| d \nu & \leq \int_{\frac{\varepsilon}{k}}^{\frac{\pi}{k}} \frac{1}{\lambda^{2}\left|D^{2}(\sigma+i \tau, \lambda)\right|}\left[1+\lambda k|\widehat{\beta}(\sigma+i \tau)|+\lambda\left|\widehat{\beta}^{\prime}(\sigma+i \tau)\right|\right] d \nu \\
& \leq \int_{\frac{\varepsilon}{k}}^{\frac{\pi}{k}} \frac{k^{2}}{(C(\varepsilon))^{2}}\left(1+\lambda k \widehat{\beta}(C(\varepsilon))+\lambda\left|\widehat{\beta}^{\prime}(C(\varepsilon))\right|\right) \\
& \leq C k \lambda,
\end{aligned}
$$

so estimate (57) holds. This proves the lemma.

3. Proof of Theorems 1 and 2. Here we adopt the overall strategy of Xu in proving our theorems, and we refer the reader to [21] for the preliminaries of the proof. We remark that $\mathrm{Xu}$ establishes the formula

$$
U^{n}(\lambda)=\operatorname{Re}\left\{\frac{1}{\pi t_{n-1} \lambda} \int_{0}^{\frac{\pi}{k}} e^{i \nu t_{n-2}} \frac{D_{s}(s(k, \nu), \lambda)}{D^{2}(s(k, \nu), \lambda)} d \nu\right\}
$$

Then, following [1], this integral is decomposed into the five parts:

$$
U^{n}(\lambda)=\operatorname{Re}\left\{\lambda^{-1} U_{1}^{n}+\lambda^{-2} U_{2}^{n}+\lambda^{-3} U_{3}^{n}+U_{4}^{n}(\lambda)+U_{5}^{n}(\lambda)\right\},
$$

where

$$
\begin{aligned}
U_{1}^{n} & =\frac{1}{\pi t_{n-1}} \int_{0}^{\varepsilon} e^{i \nu t_{n-2}} \frac{\widehat{\beta}^{\prime}(s)}{[\widehat{\beta}(s)]^{2}} d \nu, \\
U_{2}^{n} & =\frac{1}{\pi t_{n-1}} \int_{0}^{\varepsilon} e^{i \nu t_{n-2}} \frac{1}{[\widehat{\beta}(s)]^{2}}\left(1-\frac{2 s \widehat{\beta}^{\prime}(s)}{\widehat{\beta}(s)}\right) d \nu, \\
U_{3}^{n} & =\frac{-1}{\pi t_{n-1}} \int_{0}^{\varepsilon} e^{i \nu t_{n-2}} \frac{2 s}{[\widehat{\beta}(s)]^{3}} d \nu, \\
U_{4}^{n}(\lambda) & =\frac{1}{\pi t_{n-1 \lambda^{3}}} \int_{0}^{\varepsilon} e^{i \nu t_{n-2}} \frac{s^{2} D_{s}(s, \lambda)}{[\widehat{\beta}(s)]^{2} D(s, \lambda)}\left(\frac{2}{\widehat{\beta}(s)}+\frac{1}{D(s, \lambda)}\right) d \nu, \\
U_{5}^{n}(\lambda) & =\frac{1}{\pi t_{n-1 \lambda}} \int_{\varepsilon}^{\frac{\pi}{k}} e^{i \nu t_{n-2}} \frac{D_{s}(s, \lambda)}{[D(s, \lambda)]^{2}} d \nu .
\end{aligned}
$$

For $m>2$, we will establish the estimates

$$
\left|U_{4}^{n}(\lambda)\right| \leq C t_{n-2}^{-2}, \quad n \geq m, \quad \lambda \geq \lambda_{0}
$$

and either

$$
\left|U_{5}^{n}(\lambda)\right| \leq C t_{n-2}^{-2} \lambda, \quad n \geq m, \quad \lambda \geq \lambda_{0}
$$


or

$$
\left|U_{5}^{n}(\lambda)\right| \leq C t_{n-2}^{-2}, \quad n \geq m, \quad \lambda \geq \lambda_{0}
$$

to prove Theorem 1 or 2 , respectively. This follows, as then we could insert three different values of $\lambda$ into (76) and, by utilizing (53), solve for the $U_{j}^{n}(j=1,2,3)$ to show

$$
k \sum_{n=m+1}^{\infty}\left(\left|\operatorname{Re}\left\{U_{1}^{n}\right\}\right|+\left|\operatorname{Re}\left\{U_{2}^{n}\right\}\right|+\left|\operatorname{Re}\left\{U_{3}^{n}\right\}\right|\right)<\infty .
$$

Then, (34) or (35) follow, giving us Theorem 1 and 2, respectively.

We show (82) first. Integrating (80) and (81) by parts, we obtain

$$
\begin{aligned}
& U_{4}^{n}(\lambda)=\left.\frac{1}{i \pi t_{n-2} t_{n-1} \lambda^{3}} e^{i \nu t_{n-2}} \frac{s^{2} D_{s}(s, \lambda)}{[\widehat{\beta}(s)]^{2} D(s, \lambda)}\left(\frac{2}{\widehat{\beta}(s)}+\frac{1}{D(s, \lambda)}\right)\right|_{\nu=0} ^{\varepsilon} \\
&-\frac{1}{\pi t_{n-2} t_{n-1} \lambda^{3}} \int_{0}^{\varepsilon} e^{i \nu t_{n-3}} {\left[\frac{2 s D_{s}(s, \lambda)+s^{2} \widehat{\beta}^{\prime \prime}(s)}{[\widehat{\beta}(s)]^{2} D(s, \lambda)}\left(\frac{2}{\widehat{\beta}(s)}+\frac{1}{D(s, \lambda)}\right)\right.} \\
&-\frac{s^{2} D_{s}(s, \lambda)}{[\widehat{\beta}(s)]^{2} D(s, \lambda)} \\
&\left.\left(\frac{6 \widehat{\beta}^{\prime}(s)}{[\widehat{\beta}(s)]^{2}}+\frac{4 \widehat{\beta}^{\prime}(s)+2 \lambda^{-1}}{\widehat{\beta}(s) D(s, \lambda)}+\frac{2 D_{s}(s, \lambda)}{D^{2}(s, \lambda)}\right)\right] d \nu
\end{aligned}
$$

and

$$
\begin{aligned}
U_{5}^{n}(\lambda)= & \left.\frac{1}{i \pi t_{n-2} t_{n-1} \lambda} e^{i \nu t_{n-2}} \frac{D_{s}(s, \lambda)}{D^{2}(s, \lambda)}\right|_{\nu=\varepsilon} ^{\frac{\pi}{k}} \\
& -\frac{1}{i \pi t_{n-2} t_{n-1} \lambda} \int_{\varepsilon}^{\frac{\pi}{k}} e^{i \nu t_{n-3}}\left[\frac{\widehat{\beta}^{\prime \prime}(s)}{D^{2}(s, \lambda)}-\frac{2 D_{s}^{2}(s, \lambda)}{D^{3}(s, \lambda)}\right] d \nu .
\end{aligned}
$$

We see by (41) and (48) that for $0<\nu \leq \varepsilon$ with $\varepsilon$ appropriately small,

$$
|\widehat{\beta}(s)|=\sqrt{\phi^{2}(\sigma, \tau)+\tau^{2} \theta^{2}(\sigma, \tau)} \geq C \sqrt{\phi^{2}(\tau)+\tau^{2} \theta^{2}(\tau)}=C|\widehat{\beta}(i \tau)| .
$$

Then, (36), (37), (58), (63), and (87) imply that the boundary term in (85) vanishes at $\nu=0$. Note that $[1$, eq. (5.3)] and (37) give us, for $\sigma, \tau>0$,

$$
\begin{aligned}
\left|\widehat{\beta}^{\prime \prime}(s)\right| & =\mid \frac{d^{2}}{d \tau^{2}}\left(e^{-\sigma t} \beta(t) \Gamma(\tau) \mid \leq C \int_{0}^{\frac{1}{\tau}} t^{2} e^{-\sigma t} \beta(t) d t\right. \\
& \leq \frac{C}{\tau} \int_{0}^{\frac{1}{\tau}} t e^{-\sigma t} \beta(t) d t \leq \frac{C}{\tau} \theta(\tau) .
\end{aligned}
$$

Then, (36), (37), (58), (63), (87), and (88) allow us to establish the estimate (82) in a manner similar to (5.6) of [21].

To establish (83) and (84), we first note that as $\int_{0}^{\frac{2}{\sigma}} t e^{-\sigma t} d t \geq \int_{\frac{2}{\sigma}}^{\infty} t e^{-\sigma t} d t$ for all $\sigma>0$, it follows from the decreasing nature of $\beta(t)$ and (36) that

$$
\begin{aligned}
\left|\widehat{\beta}^{\prime}(\sigma)\right| & =\left(\int_{0}^{\frac{2}{\sigma}}+\int_{\frac{2}{\sigma}}^{\infty}\right) t e^{-\sigma t} \beta(t) d t \leq 2 \int_{0}^{\frac{2}{\sigma}} t e^{-\sigma t} \beta(t) d t \\
& \leq \frac{4}{\sigma} \int_{0}^{\frac{2}{\sigma}} e^{-\sigma t} \beta(t) d t \leq \frac{C}{\sigma} \widehat{\beta}(\sigma) .
\end{aligned}
$$

Copyright $\odot$ by SIAM. Unauthorized reproduction of this article is prohibited. 
Also, as $\int_{0}^{\frac{1}{\tau}} e^{-\tau t} d t \geq \int_{\frac{1}{\tau}}^{\infty} e^{-\tau t} d t$ for all $\tau>0$, we see by the decreasing nature of $\beta(t),(36)$, (42), (58), and (60) that, for $0<\sigma \leq \varepsilon \tau<\tau$,

$$
\begin{aligned}
|D(s, \lambda)| & \geq C|\widehat{\beta}(\sigma+i \tau)| \geq C \int_{0}^{\frac{1}{\tau}} e^{-\sigma t} \beta(t) d t \geq C \int_{0}^{\frac{1}{\tau}} e^{-\tau t} \beta(t) d t \\
& \geq C_{0}\left(\int_{0}^{\frac{1}{\tau}}+\int_{\frac{1}{\tau}}^{\infty}\right) e^{-\tau t} \beta(t) d t=C \widehat{\beta}(\tau) .
\end{aligned}
$$

Then, by (89) and (90), we are able to estimate the boundary terms in (86) as on pp. 148-149 of [21]. From this we obtain

$$
\left|U_{5}^{n}(\lambda)\right| \leq C t_{n-2}^{-2}\left[k^{2}+\lambda^{-1}+\lambda^{-1}\left(\int_{\varepsilon}^{\frac{\pi}{k}} \frac{\left|\widehat{\beta}^{\prime \prime}(s)\right|}{\left|D^{2}(s, \lambda)\right|}+\frac{\left|D_{s}^{2}(s, \lambda)\right|}{\left|D^{3}(s, \lambda)\right|} d \nu\right)\right] .
$$

We decompose the interval of integration into the three intervals $E_{1}=\left[\varepsilon, \frac{\varepsilon}{k}\right), E_{2}=$ $\left[\frac{\varepsilon}{k}, \frac{\pi-\varepsilon}{k}\right)$, and $E_{3}=\left[\frac{\pi-\varepsilon}{k}, \frac{\pi}{k}\right)$. To estimate the integral on $E_{1}$, we note that when $\nu \in E_{1}$, we have $0<\sigma \leq \varepsilon \tau<\tau$. So by (17), (37), (41), (47), (63), (65), (66), and (88), it follows that

$$
\begin{gathered}
\lambda^{-1} \int_{\varepsilon}^{\frac{\varepsilon}{k}}\left(\frac{\left|\widehat{\beta}^{\prime \prime}(s)\right|}{|D(s, \lambda)|^{2}}+\frac{\left|D_{s}(s, \lambda)\right|^{2}}{|D(s, \lambda)|^{3}}\right) d \nu \\
\leq C \lambda^{-1}\left(\int_{\frac{\varepsilon}{2}}^{\frac{\omega}{2}} \frac{\lambda^{-2}+\theta^{2}(\tau)}{\tau^{3} \theta^{3}(\tau)} d \tau+\int_{\frac{\omega}{2}}^{2 \omega} \frac{\lambda^{-1}}{\tau \phi^{2}(\tau)} d \tau+\int_{\frac{\omega}{2}}^{2 \omega} \frac{\lambda^{-2}}{\phi^{3}(\tau)} d \tau\right. \\
\left.\quad+\int_{2 \omega}^{\infty} \frac{\lambda d \tau}{\tau|\tau-\omega|^{2}}+\int_{2 \omega}^{\infty} \frac{\lambda d \tau}{|\tau-\omega|^{3}}\right) .
\end{gathered}
$$

Then, by (37), (46), (47), and either (17) or (18), we obtain

$$
\lambda^{-1} \int_{\varepsilon}^{\frac{\varepsilon}{k}}\left(\frac{\left|\widehat{\beta}^{\prime \prime}(s)\right|}{|D(s, \lambda)|^{2}}+\frac{\left|D_{s}(s, \lambda)\right|^{2}}{|D(s, \lambda)|^{3}}\right) d \nu \leq C(\lambda+1)
$$

or

$$
\lambda^{-1} \int_{\varepsilon}^{\frac{\varepsilon}{k}}\left(\frac{\left|\widehat{\beta}^{\prime \prime}(s)\right|}{|D(s, \lambda)|^{2}}+\frac{\left|D_{s}(s, \lambda)\right|^{2}}{|D(s, \lambda)|^{3}}\right) d \nu \leq C
$$

respectively.

To estimate the integrals on $E_{2}$ and $E_{3}$, we first note that as $\int_{0}^{\frac{3}{\sigma}} t^{2} e^{-\sigma t} d t \geq$ $\int_{\frac{3}{\sigma}}^{\infty} t^{2} e^{-\sigma t} d t$ for all $\sigma>0$, the decreasing nature of $\beta(t)$ gives us, for $\sigma>0$,

$$
\begin{aligned}
\left|\widehat{\beta}^{\prime \prime}(s)\right| & =\left|\int_{0}^{\infty} t^{2} e^{-i \tau t}\left(e^{-\sigma t} \beta(t)\right) d t\right| \leq \int_{0}^{\infty} t^{2} e^{-\sigma t} \beta(t) d t=\widehat{\beta}^{\prime \prime}(\sigma) \\
& =\left(\int_{0}^{\frac{3}{\sigma}}+\int_{\frac{3}{\sigma}}^{\infty}\right) t^{2} e^{-\sigma t} \beta(t) d t \leq 2 \int_{0}^{\frac{3}{\sigma}} t^{2} e^{-\sigma t} \beta(t) d t \\
& \leq \frac{18}{\sigma^{2}} \int_{0}^{\frac{3}{\sigma}} e^{-\sigma t} \beta(t) d t \leq \frac{C}{\sigma^{2}} \widehat{\beta}(\sigma) .
\end{aligned}
$$

Copyright $@$ ㅇ by SIAM. Unauthorized reproduction of this article is prohibited. 
Then, in the case where $\nu \in E_{2}$, we see that $\sigma \leq \frac{2}{k}$ and $\tau \geq \frac{\sin (\varepsilon)}{k}$. Then (39) and (40) give, for $\nu \in E_{2}$,

$$
\begin{aligned}
\phi(\sigma, \tau) & \geq-C \int_{0}^{\frac{1}{\tau}} t e^{-\sigma t} \beta^{\prime}(t) d t \geq-C e^{\frac{-\sigma}{\tau}} \int_{0}^{\frac{1}{\tau}} t \beta^{\prime}(t) d t \\
& \geq-C e^{\frac{-2}{\sin (\varepsilon)}} \int_{0}^{\frac{1}{\tau}} t \beta^{\prime}(t) d t \geq C \phi(\tau) .
\end{aligned}
$$

So, we see that (17), (63), (88), and (96) give us

$$
\begin{aligned}
& \lambda^{-1} \int_{\frac{\varepsilon}{k}}^{\frac{\pi-\varepsilon}{k}} \frac{\left|\widehat{\beta}^{\prime \prime}(s)\right|}{\left|D^{2}(s, \lambda)\right|}+\frac{\left|D_{s}^{2}(s, \lambda)\right|}{\left|D^{3}(s, \lambda)\right|} d \nu \\
& \quad \leq C \lambda^{-1} \int_{\frac{\varepsilon}{k}}^{\frac{\pi-\varepsilon}{k}} \frac{k \lambda \theta(\tau)}{\tau \phi(\tau)}+k^{3} \lambda+\frac{k^{2} \lambda \theta(\tau)}{\phi(\tau)}+\frac{k \lambda \theta^{2}(\tau)}{\phi^{2}(\tau)} d \nu \\
& \quad \leq C .
\end{aligned}
$$

For the integral on $E_{3}$, note that since we have $\frac{2-\varepsilon}{k} \leq \sigma \leq \frac{2}{k}$ and $0 \leq \tau \leq \frac{\varepsilon}{k}$ for $\nu \in E_{3}$, we see by (52), (89), and (95) that

$$
\begin{aligned}
& \lambda^{-1} \int_{\frac{\pi-\varepsilon}{k}}^{\frac{\pi}{k}} \frac{\left|\widehat{\beta}^{\prime \prime}(s)\right|}{\left|D^{2}(s, \lambda)\right|}+\frac{\left|D_{s}^{2}(s, \lambda)\right|}{\left|D^{3}(s, \lambda)\right|} d \nu \\
& \quad \leq C \lambda^{-1} \int_{\frac{\pi-\varepsilon}{k}}^{\frac{\pi}{k}} \frac{\lambda \widehat{\beta}(\sigma)}{\sigma^{3} \widehat{\beta}(\sigma)}+\frac{\lambda}{\sigma^{3}}+\frac{\lambda \widehat{\beta}(\sigma)}{\sigma^{3} \widehat{\beta}(\sigma)}+\frac{\lambda[\widehat{\beta}(\sigma)]^{2}}{\sigma^{3}[\widehat{\beta}(\sigma)]^{2}} d \nu \\
& \quad \leq C .
\end{aligned}
$$

Then, by (97), (98), and either (93) or (94), we have established (83) or (84), respectively, and thus we have proven Theorems 1 or 2, respectively.

\section{REFERENCES}

[1] R. W. Carr And K. B. Hannsgen, A nonhomogeneous integrodifferential equation in Hilbert space, SIAM J. Math. Anal., 10 (1979), pp. 961-984.

[2] R. W. CARR AND K. B. Hannsgen, Resolvent formulas for a Volterra equation in Hilbert space, SIAM J. Math. Anal., 13 (1982), pp. 459-483.

[3] E. Cuesta, C. Lubich, And C. Palencia, Convolution quadrature time discretization of fractional diffusive-wave equations, Math. Comp., 75 (2006), pp. 673-696.

[4] P. L. Duren, The Theory of $H^{p}$ Spaces, Academic, New York, 1970.

[5] G. Gripenberg, S-O. Londen, and O. Staffens, Volterra Integral and Functional Equations, Cambridge University Press, Cambridge, 1990.

[6] K. B. Hannsgen, Indirect Abelian theorems and a linear Volterra equation, Trans. Amer. Math. Soc., 142 (1969), pp. 539-555.

[7] C. Lubich, Convolution quadrature and discretized operational calculus I, Numer. Math., 52 (1988), pp. 129-145

[8] C. Lubich, I. H. Sloan, And V. ThoméE, Nonsmooth data error estimates for approximations of an evolution equation with a positive-type memory term, Math. Comp., 65 (1996), pp. 117 .

[9] W. Mclean and K. Mustapha, A second-order accurate numerical method for a fractional wave equation, Numer. Math., 105 (2007), pp. 481-510.

[10] W. MCLean And V. Thomée, Numerical solution of an evolution equation with a positive type memory term, J. Aust. Math. Soc., 35 (1993), pp. 23-70.

[11] W. McLean and V. Thomee, Numerical solution via Laplace transforms of a fractional order evolution equation, J. Integral Equations Appl., 22 (2010), pp. 57-94.

Copyright (c) by SIAM. Unauthorized reproduction of this article is prohibited. 
[12] K. Mustapha And W. McLean, Discontinuous Galerkin method for an evolution equation with a memory term of positive type, Math. Comp., 78 (2009), pp. 1975-1995.

[13] J. A. Nohel And D. F. SheA, Frequency domain methods for Volterra equations, Adv. Math., 22 (1976), pp. 278-304

[14] R. D. Noren, Uniform $L^{1}$ behavior for the solution of a Volterra equation with a parameter, SIAM J. Math. Anal., 19 (1988), pp. 270-286.

[15] J. Prüss, Evolutionary Integral Equations and Applications, Monogr. Math. 87, Birkhäuser, Basel, 1993.

[16] M. Renardy, W. J. Hrusa, and J. A. Nohel, Mathematical Problems in Viscoelasticity, Longman, London, 1987.

[17] F. Riesz And B. Sz.-Nagy, Functional Analysis, Dover, New York, 1990.

[18] W. Rudin, Real and Complex Analysis, McGraw-Hill, Boston, 1987.

[19] D. F. SheA And S. Wainger, Variants of the Wiener-Levy theorem with applications to stability problems for some Volterra integral equations, Amer. J. Math., 97 (1975), pp. 312-343.

[20] D. V. Widder, The Laplace Transform, Princeton University Press, Princeton, NJ, 1946.

[21] D. Xu, Uniform $l^{1}$ behavior for time discretization of a Volterra equation with completely monotonic kernel: I. Stability, IMA J. Numer. Anal., 22 (2002), pp. 133-151.

[22] D. XU, Uniform $l^{1}$ behavior for time discretization of a Volterra equation with completely monotonic kernel: II. Convergence, SIAM J. Numer. Anal., 46 (2008), pp. 231-259.

[23] D. Xu, Stability of the difference type methods for linear Volterra equations in Hilbert space, Numer. Math., 109 (2008), pp. 571-595.

[24] D. Xu, Uniform $l^{1}$ error bounds for the semidiscrete solution of a Volterra equation with completely monotonic convolution kernel, Comput. Math. Appl., 43 (2002), pp. 1303-1318.

Copyright $@$ by SIAM. Unauthorized reproduction of this article is prohibited. 